\title{
Serviço de Cirurgia Fetal da Universidade Federal do Ceará - breve histórico
}

A Medicina Fetal é uma especialidade que representa um conjunto de ações de finalidades preventivas, diagnósticas e terapêuticas, no sentido de proteger, avaliar e assistir a saúde do feto. São exigidos conhecimentos fundamentais de embriologia, obstetrícia, genética médica, neonatologia, morfologia, dismorfologia, fisiologia e fisiopatologia fetais. Os procedimentos terapêuticos vão desde punções de derrames em cavidades fetais até cirurgias corretivas ainda no ambiente intrauterino; portanto, pressupõe necessidade de equipe multiprofissional altamente especializada para êxito materno e fetal seguindo indicações precisas após conhecimento da evolução natural das patologias com respeito aos preceitos da ética médica.

No ano de 1991 os Profs. Drs. Carlos Augusto Alencar Junior e Francisco Manuelito de Almeida iniciam o Serviço de Medicina Materno-Fetal na Maternidade Escola Assis Chateaubriand-UFC com dois setores: (1) O ambulatório especializado em Gestação de Risco, conduzido principalmente por Ana Paula Andrade Augusto e (2) Serviço de Medicina Fetal com suporte de imagem para acompanhamento das gestações, operacionalizado inicialmente pelo próprio Carlos Augusto e Maria do Carmo da Mota Seabra. Esse serviço já inicia com parceria simbiótica do Serviço de Neonatologia e de outros profissionais, entre eles psicólogos e assistentes sociais.

Desde então o serviço se responsabiliza por procedimentos diagnósticos e terapêuticos de várias patologias maternas e fetais. Na primeira fase de implantação os diagnósticos se restringiam à ultrassonografia. Posteriormente foram introduzidos os procedimentos diagnósticos de cordocentese, amniocentese e biópsia de vilosidades coriônicas e especialmente as transfusões intrauterinas para gestações acometidas com isoimunização pelo fator $\mathrm{Rh}$ - procedimento salvador de muitas vidas nessa patologia ainda prevalente, apesar da existência de profilaxia primária.

Muitos outros profissionais vieram a ser incorporados ao serviço: Especialistas em Ultrassonografia em Ginecologia e Obstetrícia (José de Arimatea Barreto, Julio Augusto G. Alves, Raimundo Homero de Carvalho Neto, Pollyana Maria F. S. Barbosa) e Medicina Fetal (Helvécio Neves Feitosa, Virlenia Paula B. Oliveira, Eliana M. Tomaz C. Sales, Lilian M. Fechine Belchior e Henry Wong Vela). Profissionais de outras áreas foram incorporados ao Serviço: Geneticistas, Cirurgiões Pediátricos, Neurocirurgiões, Radiologistas, Biólogos e Biomédicos.

O Conselho Federal de Medicina (CFM) reconhece a Medicina Fetal como área de atuação e a FEBRASGO (Federação Brasileira das Sociedades de Ginecologia e Obstetrícia) em conjunto com a AMB passou a expedir título de especialista no ano de 2004, quando foram titulados os primeiros especialistas do Ceará: Carlos Augusto, Edson Lucena, Herlânio Costa e Helvécio Neves Feitosa. Em 2015, o Complexo Hospitalar da UFC iniciou a Residência Médica em Medicina Fetal, sob coordenação do Prof. Dr. Herlânio Costa.

Nos últimos 15 anos a especialidade alcançou evolução muito grande com procedimentos cada vez mais complexos. Havia a necessidade de alçarmos outros voos. A equipe buscou conhecimento em outras instituições nacionais, especialmente na UNIFESP (serviço chefiado pelo Prof. Antonio Fernandes Moron, responsável pela primeira cirurgia a céu aberto para mielomeningocele no Brasil) e internacionais: Universidade de Barcelona (serviço do Prof. Eduard Gratacós), Kings College of London (serviço do Prof. Kypros Nicolaides), Cincinnati Children's Hospital (Prof. Jose L. Peiró), entre outros.

Em paralelo ao desejo de evoluir nas intervenções fetais, foram realizados entre 2009 e 2014 na Faculdade de Medicina da Universidade Federal do Ceará (Famed-UFC), projetos de pesquisa experimentais visando melhor compreensão da fisiopatologia de malformações congênitas fetais. Os projetos foram desenvolvidos sob a coordenação do Prof. Antônio Aldo Melo Filho (Departamento de Cirurgia) com a orientação do Prof. Lourenço Sbragia (FMRP-USP) que já era um dos principais pesquisadores internacionais na área. Para sua execução, foram desenvolvidas parcerias com vários docentes pesquisadores dos Departamentos de Fisiologia e Farmacologia e Departamento de Morfologia da UFC. Entre estes, registramos a atuação decisiva dos Profs. Drs. Armênio Aguiar dos Santos, Ronaldo de Albuquerque Ribeiro (in memoriam), Pedro Jorge Caldas Magalhães, Marcellus Henrique Loiola Ponte de Souza e Gerly Anne de Castro Brito.

Neste período, se destacaram estudos experimentais envolvendo, notadamente, duas doenças congênitas fetais importantes passíveis de diagnóstico fetal e tratamento cirúrgico pós-natal: gastrosquise e atresia de esôfago. Foram eles: estudo das repercussões inflamatórias no trato digestório de fetos com gastrosquise e estudo de patogênese e repercussões na motilidade do trato digestório em fetos com atresia de esôfago, executados com financiamento do CNPq. Além de diversos trabalhos apresentados em congressos locais, nacionais e internacional, esses produziram uma publicação no periódico científico oficial da Academia Americana de Cirurgia Pediátrica e no principal periódico científico brasileiro, com penetração internacional, sobre pesquisa experimental. Um deles recebeu ainda o Prêmio "Prof. Lacerda Machado" de melhor trabalho experimental na XIX 
Jornada Anual de Cirurgia, em 2011, promovida anualmente pelo Departamento de Cirurgia da UFC. Além do envolvimento de alunos de iniciação científica, uma dissertação de Mestrado e uma tese de doutorado foram concluídas na Famed-UFC com tais pesquisas.

Ainda entre 2009 e 2012, foi desenvolvido um estudo clínico "Estratégias para redução de morbi-mortalidade materna e perinatal, em gestações com malformações congênitas", sob a coordenação geral também do Prof. Aldo Melo e financiamento da FUNCAP. Este projeto foi uma das primeiras tentativas de realizar procedimentos fetais a céu aberto na UFC. Apesar de não ter obtido êxito em realizar o procedimento em si, desencadeou intensa discussão entre as áreas de Obstetrícia, Cirurgia Pediátrica, Neonatologia e Anestesiologia e produziu dados sobre a epidemiologia das mães gestantes com filhos com malformações congênitas atendidas na MEAC. Um dos seus sub-projetos foi executado em parceria com a Profa. Dra. Márcia Maria Tavares Machado (atual PróReitora de Extensão da UFC). Neste foi realizado um estudo qualitativo que evidenciou a repercussão negativa do diagnóstico fetal de malformação (mesmo quando leve) para o vínculo materno-fetal e vida social da mulher e a necessidade de atuação multidisciplinar para minimizar este impacto e acolher a família.

Nessa mesma época, o Neurocirurgião Pediátrico Eduardo Jucá também atuava em estudos experimentais de cirurgia fetal para mielomeningocele sob a orientação do Prof. Lourenço Sbraggia na USP em Ribeirão Preto e posteriormente em Paris em projeto associado ao serviço de Neurocirurgia Pediátrica do Hospital Necker. A formação específica em Neurocirurgia Pediátrica e o interesse continuado em cirurgia fetal o levaram ainda a visitar o serviço dos Profs. Antônio Moron e Sérgio Cavalheiro já em 2016.

O resultado de todos estes projetos foi, no entanto, mais além. Eles colaboraram para disseminar a importância de estudar o feto e suas doenças entre alunos e docentes da Famed-UFC, em uma extensa rede multidisciplinar que é o "DNA" da Medicina Fetal. Eles ainda colaboraram para fortalecer o ensino de graduação, estreitando as áreas básica e clínica entre os Departamentos de Morfologia, Saúde Materno Infantil (hoje Saúde da Mulher, da Criança e do Adolescente) e Cirurgia. Destacam-se aqui as atuações da Profas. Dras. Renata Ferreira de Carvalho Leitão e Virgínia Cláudia Carneiro Girão. Um dos outros resultados recentes da colaboração a este desenvolvimento foi o exitoso I Simpósio de Embriologia 4D que ocorreu nos dias 17 e 18 de maio de 2019. O evento foi uma iniciativa do Programa de Pós-Graduação em Ciências Morfofuncionais (Departamento de Morfologia - Famed - UFC) e da Seara da Ciência (UFC), tendo sido coordenado pela Profa. Virgínia Girão. Revelou a formidável e importante integração entre as áreas básicas e clínicas. Entre outros, palestraram neste evento, os Profs. Drs. Herlânio Costa, Aldo Melo e Eduardo Jucá que viriam a atuar na primeira cirurgia fetal na MEAC-UFC dias depois, juntamente com Edson Lucena.

A primeira cirurgia fetal para correção intraútero de Mielomeningocele no Ceará foi possível graças à colaboração de vários profissionais além dos já citados: anestesia, equipes de enfermagem, farmácia hospitalar e engenharia clínica. Nesse primeiro procedimento contou-se com o suporte da equipe da UNIFESP (Profs. Drs. Antonio Moron, Mauricio Mendes Barbosa, Sérgio Cavalheiro e Ítalo Suriano).

A Mielomeningocele (MMC) é a primeira anomalia não-letal considerada para intervenção cirúrgica fetal. Estudos recentes randomizados sugerem que a cirurgia fetal da MMC pode reduzir a necessidade de derivações ventrículo-peritoneal e melhora das condições motoras dos membros inferiores em comparação com controles operados após o nascimento. O MOMS (Management of Myelomeningocele Study) foi publicado em março de 2011. A partir dos resultados promissores do MOMS, este procedimento deixou de ser experimental estando disponível em diversos centros americanos e contando com a recomendação do Colégio Americano de Ginecologia e Obstetrícia (ACOG). Com a expectativa de um número crescente de centros terciários disponibilizarem este tipo de intervenção cirúrgica, ficou claro a necessidade de critérios mínimos para constituição destes centros para garantir os melhores resultados associado a segurança materna e fetal.

Os centros devem contar com equipe multidisciplinar, onde cada profissional tem sua função, interagindo em todos os momentos do tratamento. Os riscos maternos não devem ser negligenciados estando relacionados basicamente ao procedimento e ao uso de anestésicos, tocolíticos e outros medicamentos. A equipe cirúrgica deve ter preparo adequado, utilizando técnicas eficientes e seguras para abertura e fechamento do útero, incluindo cirurgiões com habilidade para correção de anomalias em fetos com menos de 26 semanas de gestação mantendo estáveis as condições hemodinâmicas maternas e fetais durante todo o procedimento. É fundamental dispor de infraestrutura hospitalar de nível terciário de alta complexidade e com recursos para tratamento intensivo materno e do recém-nascido.

\section{REFERÊNCIAS}

1 American College of Obstetricians and Gynecologists. ACOG Committee opinion no. 550: maternal-fetal surgery for myelomeningocele. Obstet Gynecol. 2013;121(1):218-9.

2 Adzick NS, Thom EA, Spong CY, Brock JW 3rd, Burrows PK, Johnson MP, et al. A randomized trial of prenatal versus postnatal repair of myelomeningocele. N Engl J Med. 2011;364(11):993-1004. 
3 Moron AF, Barbosa MM, Milani HJ, Hisaba W, Carvalho NS, Cavalheiro S. Short-term surgical and clinical outcomes with a novel method for open fetal surgery of myelomeningocele. Am J Obstet Gynecol. 2015;212(1):S374.

4 Cavalheiro S, da Costa MD, Moron AF, Leonard J. Comparison of prenatal and postnatal management of patients with myelomeningocele. Neurosurg Clin N Am. 2017;28(3):439-48.

Antonio Aldo de Melo Filho ${ }^{1}$. Francisco Edson de Lucena Feitosa ${ }^{1}$. Francisco Herlânio Costa Carvalho ${ }^{1}$. Carlos Eduardo Barros Jucá $^{1}$. Liliana Soares Nogueira Paes ${ }^{1}$. Intã Bruna Cerqueira Silva Tomaz ${ }^{1}$. Fernanda Oliveira de Castro ${ }^{1}$. Luciana Maria de Oliveira Nascimento ${ }^{1}$. Maria da Piedade Albuquerque ${ }^{1}$. Carlos Augusto Alencar Junior ${ }^{1}$.

1 Maternidade Escola Assis Chateaubriand (HUWC), Universidade Federal do Ceará (UFC), Fortaleza, Ceará, Brasil.

\section{Como citar:}

Melo AA Filho, Feitosa FE, Carvalho FH, Jucá CE, Paes LS, Tomaz IB, et al. Serviço de Cirurgia Fetal da Universidade Federal do Ceará - breve histórico. Rev Med UFC. 2019 abr-jun;59(2):6-8. 